\title{
Clinical implications of human leukocyte antigen class I expression in endometrial cancer
}

\author{
KAZUYUKI YAKABE, AKIHIRO MURAKAMI, YUKI NISHIMOTO, TAKUYA KAJIMURA, \\ KOTARO SUEOKA and NORIHIRO SUGINO
}

Department of Obstetrics and Gynecology, Graduate School of Medicine, Yamaguchi University, Ube, Yamaguchi 755-8505, Japan

Received March 23, 2015; Accepted May 18, 2015

DOI: $10.3892 / \mathrm{mco} .2015 .636$

\begin{abstract}
Decreased expression of human leukocyte antigen (HLA) class I molecules, which is found in several types of cancer, is associated with worse clinical prognosis in cancer patients. The present study was undertaken to investigate the association of immunohistochemical HLA class I expression patterns with clinicopathological factors and prognosis in 96 endometrial cancer patients. HLA class I is composed of a heavy chain (HC-10) and a $\beta 2$-microglobulin $(\beta 2-\mathrm{m})$ light chain. The HLA class I expression patterns were classified as positive when both $\mathrm{HC}-10$ and $\beta 2-\mathrm{m}$ were strongly stained and negative in all other cases. The negative staining pattern was associated with advanced International Federation of Gynecology and Obstetrics stage $(\mathrm{P}<0.001)$, lymphovascular space involvement (LVSI) $(\mathrm{P}=0.003)$ and lymph node metastasis $(\mathrm{P}=0.005)$. Moreover, these cases exhibited worse progression-free survival (PFS) and overall survival (OS) rates compared with positive cases $(\mathrm{P}=0.005$ and $\mathrm{P}=0.014$, respectively). However, the multivariate analysis did not identify HLA class I expression as an independent predictive factor for PFS and OS. In conclusion, HLA class I expression may be useful for predicting postoperative outcome in endometrial cancer, as well as well-known predictive prognostic factors, such as lymph node metastasis and LVSI.
\end{abstract}

\section{Introduction}

The immune system plays a role in controlling tumor growth and tumor cell metastasis. Human leukocyte antigen (HLA) class I molecules are transmembrane glycoproteins comprising a heavy chain (HC-10) and a $\beta 2$-microglobulin $(\beta 2-\mathrm{m})$ light

Correspondence to: Professor Norihiro Sugino, Department of Obstetrics and Gynecology, Graduate School of Medicine, Yamaguchi University, 1-1-1 Minamikogushi, Ube, Yamaguchi 755-8505, Japan E-mail: sugino@yamaguchi-u.ac.jp

Key words: endometrial cancer, immunohistochemistry, human leukocyte antigen class I heavy chain, $\beta 2$-microglobulin, prognostic factors chain. These antigens are expressed on the surface of the tumor cells and their presence is considered to be a prerequisite for an effective T-cell immune response $(1,2)$. Therefore, tumor cells with downregulated HLA class I expression escape this immune response, resulting in an increase in growth or an increased ability to invade other organs (3).

Downregulation of HLA class I expression occurs in a variety of cancers and is associated with a poor prognosis (4-11). Determining HLA class I expression may prove useful for the prediction of tumor progression and recurrence risk via the antitumor immune system $(6,7)$. However, only a limited number of studies have investigated the clinical implication of HLA class I expression in endometrial cancer. The degree of HLA class I expression was found to be correlated with disease stage (12), but that study lacked a multivariate analysis. Patients in whom HLA class I expression was downregulated had a lower disease-specific survival compared with patients exhibiting normal expression (13). On the other hand, HLA class I expression was not found to be associated with clinicopathological parameters or survival (10). Further studies are required to elucidate whether HLA class I expression is of value as a predictive prognostic factor in endometrial cancer. The present study was undertaken to investigate the association of immunohistochemical HLA class I expression patterns with clinicopathological factors and prognosis in endometrial cancer.

\section{Materials and methods}

Patients and specimens. We investigated 96 patients who underwent hysterectomy with pelvic and paraaortic lymphadenectomy for endometrial cancer at the Yamaguchi University Hospital(Ube,Japan). The pathological diagnosis of the samples was performed by specialized gynecological pathologists. None of the patients had received anticancer chemotherapy or radiation therapy prior to surgery. All the tumor tissues were obtained from surgically resected specimens.

This study was reviewed and approved by the Institutional Review Board of Yamaguchi University Graduate School of Medicine.

Immunohistochemistry. The streptavidin-biotin-peroxidase complex technique was used for immunohistochemistry, as 
A
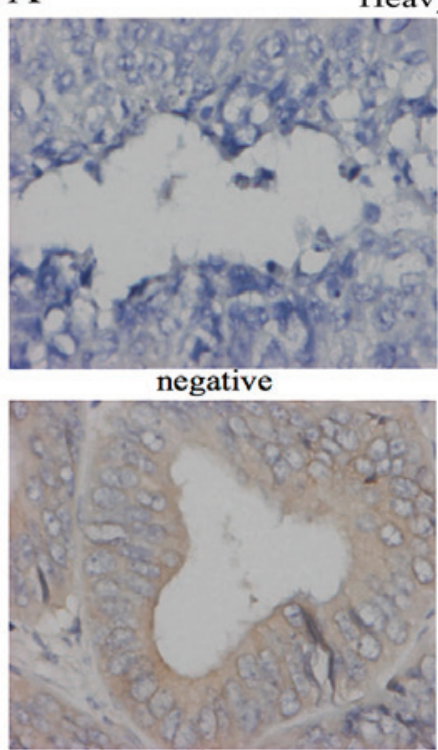

moderate
Heavy chain

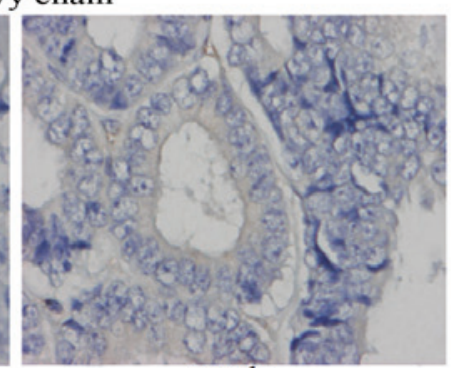

weak

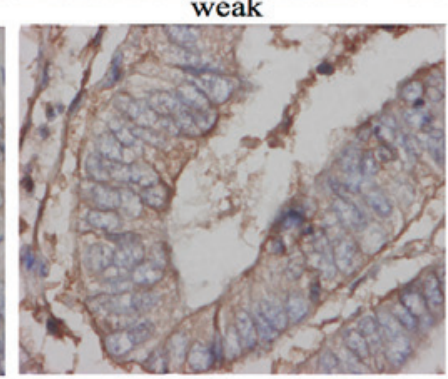

strong
B
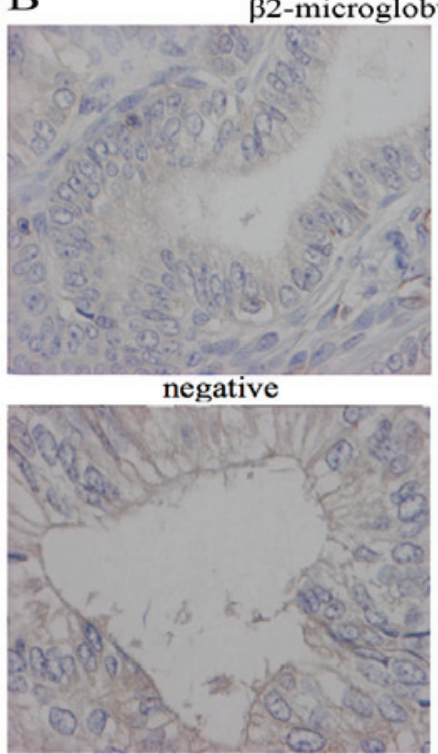

moderate $\beta 2$-microglobulin light chain
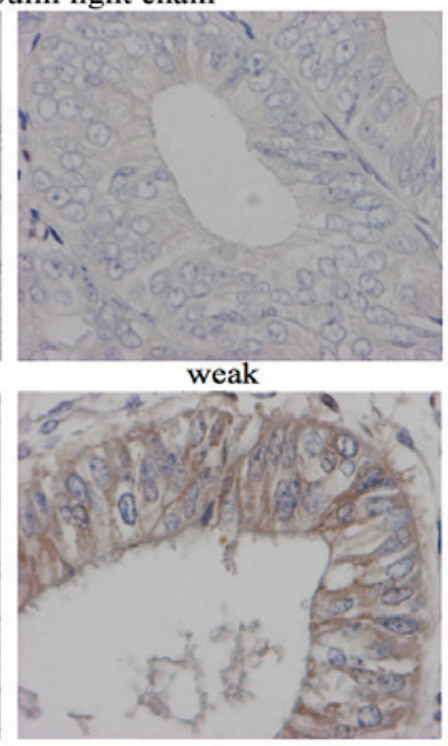

strong

Figure 1. Representative endometrial cancer sections immunohistochemically stained for (A) heavy chain (HC-10) and (B) $\beta 2$-microglobulin light chain $(\beta 2-\mathrm{m})$. The staining intensity was divided into four patterns, namely negative, weak, moderate and strong.

previously described (14). In brief, the specimens were fixed in $10 \%$ buffered formalin and embedded in paraffin. All the paraffin blocks were cut into 3- $\mu \mathrm{m}$ sections for immunohistochemistry. A series of sections were deparaffinized in xylene and rehydrated in a graded ethanol series. The samples were washed with cold phosphate-buffered saline (PBS). Endogenous peroxidase was blocked by incubating the sections with $0.5 \%$ hydrogen peroxide in methanol for $50 \mathrm{~min}$ at room temperature. The sections were then washed three times in cold PBS. Antigen retrieval was performed by incubating the sections in heated antigen retrieval solution (Nacalai Tesque Inc., Kyoto, Japan) for $30 \mathrm{~min}$ at $100^{\circ} \mathrm{C}$ using a water bath, followed by washing three times in cold PBS. Following incubation with either $10 \%$ normal rabbit serum for HC-10 or $10 \%$ normal goat serum for $\beta 2$-m to block non-specific binding, the specimens were sequentially incubated with the primary antibody at $4^{\circ} \mathrm{C}$ overnight. The following primary antibodies were used: Mouse anti-human monoclonal antibody to HC-10 (EMR 8-5; Hokudo, Sapporo, Japan) and rabbit anti-human polyclonal antibody to 32-m(EMR B-6; Dako, Copenhagen, Denmark) (15). Following incubation, the sections were rinsed in cold PBS and incubated for $10 \mathrm{~min}$ at room temperature with biotinylated anti-mouse $\operatorname{IgG}+\operatorname{Ig} \mathrm{A}+\operatorname{IgM}$ to HC-10 or anti-rabbit $\operatorname{IgG}$ to $\beta 2$-m using the Histofine SAB-PO kit (Nichirei, Tokyo, Japan), followed by the streptavidin-peroxidase complex. The reaction was visualized by adding diaminobenzidine tetrahydrochloride chromogen mixture (Sigma, St. Louis, MO, USA). Following hematoxylin counterstaining, the slides were permanently mounted and analyzed for the presence and distribution of immunostaining. The results were assessed in a blinded fashion by two independent observers.

Evaluation of HLA class I expression. HC-10 and $\beta 2-\mathrm{m}$ expression was evaluated according to the method described by Murakami et al (16). Since HLA class I is usually expressed on the surface of cancer cells, the staining intensity on cancer cell membranes was evaluated. A score was established corresponding to the sum of i) the percentage of positive cells $(0,0 \%$ immunopositive cells; $1,<50 \%$ positive cells; and $2,>50 \%$ positive cells); and ii) the staining intensity ( 0 , absent; 1, weak; 2, moderate; and 3, strong). Representative examples of staining intensity are shown in Fig. 1. The sum of assigned values of the positive cell percentage and the staining intensity was calculated and used to identify three categories of expression: Scores of 0 were considered as loss, scores of 2 and 3 as weak staining and scores of 4 and 5 as strong staining. The HLA class I expression patterns were classified into two groups (positive and negative) according to the method described by Rolland et al (4). Since HLA class I expression is composed of $\mathrm{HC}-10$ and $\beta 2-\mathrm{m}$ expression, the cases in which both HC-10 and $\beta 2-\mathrm{m}$ were strongly stained were defined as HLA class I-positive; any deviation from that pattern was defined as HLA class I-negative.

Statistical analysis. The association between HLA class I positivity and negativity and clinicopathological parameters was analyzed by the Chi-square distribution. Progression-free survival (PFS) was defined as the time between the date of the operation and the date of recurrence or the last date of follow-up. Overall survival (OS) was defined as the time between the date of the operation and the date of death or the last date of follow-up. The analyses of PFS and OS were based on the Kaplan-Meier method. The PFS and OS curves were compared with the log-rank test. The Cox proportional hazard analysis was used for multivariate analysis to determine the relative risk and independent significance of individual factors. $\mathrm{P}<0.05$ was considered to indicate statistically significant differences. The statistical analyses of the study data were performed using IBM SPSS statistics for Windows software version 11 (SPSS Inc., Chicago, IL, USA). 
Table I. Association between clinicopathological factors and HLA class I expression pattern $(n=96)$.

\begin{tabular}{|c|c|c|c|c|}
\hline Variables & Positive $(\mathrm{n}=51)$ & Negative $(n=45)$ & Total no. $(\%)$ & P-value \\
\hline Age (years) & & & & 0.072 \\
\hline$<50$ & 13 & 5 & $18(18.7)$ & \\
\hline$>50$ & 38 & 40 & $78(81.3)$ & \\
\hline FIGO $^{a}$ stage & & & & $<0.001$ \\
\hline I & 48 & 29 & $77(80.2)$ & \\
\hline II & 1 & 2 & $3(3.1)$ & \\
\hline III & 0 & 12 & $12(12.5)$ & \\
\hline IV & 2 & 2 & $4(4.2)$ & \\
\hline Myometrial invasion & & & & 1.000 \\
\hline Depth $\mathrm{a}^{\mathrm{a}}$ & 34 & 30 & $64(66.7)$ & \\
\hline Depth $b^{b}$ & 17 & 15 & $32(33.3)$ & \\
\hline LVSI & & & & 0.003 \\
\hline Positive & 12 & 24 & $36(37.5)$ & \\
\hline Negative & 39 & 21 & $60(62.5)$ & \\
\hline Tumor grade & & & & 0.051 \\
\hline 1 & 30 & 16 & $46(47.9)$ & \\
\hline 2 & 19 & 22 & $41(42.7)$ & \\
\hline 3 & 2 & 7 & $9(9.4)$ & \\
\hline Lymph node metastasis & & & & 0.005 \\
\hline Positive & 1 & 8 & $9(9.4)$ & \\
\hline Negative & 50 & 33 & $83(86.4)$ & \\
\hline Missing & & & $4(4.2)$ & \\
\hline Peritoneal cytology & & & & 0.211 \\
\hline Positive & 9 & 4 & $13(13.6)$ & \\
\hline Negative & 42 & 41 & $83(86.4)$ & \\
\hline
\end{tabular}

Bold print indicates statistical significance. Invasion of aless than half and ${ }^{\mathrm{b}}$ more than half of the thickness of the myometrium. HLA, human leukocyte antigen; FIGO, International Federation of Gynecology and Obstetrics; LVSI, lymphovascular space involvement.

Table II. Evaluation of HLA class I expression.

\begin{tabular}{lccr}
\hline HLA class I expression & HC-10 & $\beta 2-\mathrm{m}$ & No. \\
\hline Positive & + & + & 51 \\
\multirow{2}{*}{ Negative } & + & - & 13 \\
& - & + & 9 \\
& - & - & 23
\end{tabular}

The sum of assigned values of the percentage of positive cells $(0,0 \% ; 1,<50 \%$; and $2,>50 \%$ positive cells) and the staining intensity ( 0 , absent; 1 , weak; 2 , moderate; and 3, strong) was used to identify three categories of expression: Scores of 0 were considered as loss (-); 2 and 3, weakly stained (-); and 4 and 5, strongly stained (+). HLA, human leukocyte antigen; HC-10, heavy chain; $\beta 2-\mathrm{m}, \beta 2$-microgloblin light chain.

\section{Results}

Clinicopathological characteristics. The clinicopathological characteristics of the patients are summarized in Table I.
The median age at diagnosis was 56 years and the median follow-up duration was 45 months (range, 1-178 months). Of the 96 patients, 77 had stage I $(80.2 \%), 3$ had stage II $(3.1 \%)$, 12 had stage III (12.5\%) and 4 had stage IV (4.2\%) disease, as determined by the International Federation of Gynecology and Obstetrics (FIGO). In $64(66.7 \%)$ of the patients, the tumor invaded less than half of the thickness of the myometrium (depth a). Lymphovascular space involvement (LVSI) was detected in 36 patients $(37.5 \%)$. A total of 9 patients $(9.4 \%)$ had grade 3, $41(42.7 \%)$ had grade 2 and $46(47.9 \%)$ had grade 1 tumors. Positive lymph node metastasis was identified in 9 patients $(9.4 \%)$ and the peritoneal cytology was positive in 13 patients $(13.6 \%)$.

Expression and evaluation of HLA class I molecules. As shown in Table II, 51 cases were HLA class I-positive, with strong staining for both $\mathrm{HC}-10$ and $\beta 2-\mathrm{m}$, while 45 cases were HLA class I-negative. The latter included weak staining (or loss) of either HC-10 or $\beta 2-\mathrm{m}$, or both. A univariate analysis using the Chi-square distribution revealed a significant correlation between strongly stained HC-10 and strongly stained $\beta 2-\mathrm{m}(\mathrm{P}<0.001)$. 
Table III. Cox regression analysis of progression-free survival.

\begin{tabular}{|c|c|c|c|c|}
\hline \multirow[b]{2}{*}{ Variables } & \multicolumn{2}{|c|}{ Univariate analysis } & \multicolumn{2}{|c|}{ Multivariate analysis } \\
\hline & HR $(95 \% \mathrm{CI})$ & $\mathrm{P}$-value & HR (95\% CI) & P-value \\
\hline \multicolumn{5}{|l|}{ Age (years) } \\
\hline$>50$ vs. $<50$ & $2.329(0.296-18.317)$ & 0.422 & $1.103(0.129-9.425)$ & 0.928 \\
\hline \multicolumn{5}{|l|}{ FIGO stage } \\
\hline III/IV vs. I/II & $26.192(5.613-122.21)$ & $<0.001$ & $31.294(3.728-262.69)$ & 0.002 \\
\hline \multicolumn{5}{|l|}{ Myometrial invasion } \\
\hline Depth $a^{a}$ vs. $b^{b}$ & $3.586(1.047-12.279)$ & 0.042 & - & - \\
\hline \multicolumn{5}{|l|}{ LVSI } \\
\hline Positive vs. negative & $4.579(1.205-17.401)$ & 0.026 & $0.444(0.081-2.443)$ & 0.351 \\
\hline \multicolumn{5}{|l|}{ Tumor grade } \\
\hline 3 vs. $1 / 2$ & $5.839(1.498-22.764)$ & 0.011 & $3.446(0.734-16.179)$ & 0.117 \\
\hline \multicolumn{5}{|l|}{ Lymph node metastasis } \\
\hline Positive vs. negative & $9.629(2.301-40.294)$ & 0.002 & - & - \\
\hline \multicolumn{5}{|l|}{ Peritoneal cytology } \\
\hline Positive vs. negative & $0.505(0.064-4.009)$ & 0.518 & $3.381(0.278-41.099$ & 0.339 \\
\hline \multicolumn{5}{|c|}{ HLA class I expression pattern } \\
\hline Positive vs. negative & $0.094(0.012-0.742)$ & 0.025 & $0.361(0.033-3.920)$ & 0.403 \\
\hline
\end{tabular}

Bold print indicates statistical significance. Invasion of ${ }^{a}$ less than half and ${ }^{b}$ more than half of the thickness of the myometrium. HR, hazard ratio; CI, confidence interval; FIGO, International Federation of Gynecology and Obstetrics; LVSI, lymphovascular space involvement; HLA, human leukocyte antigen.

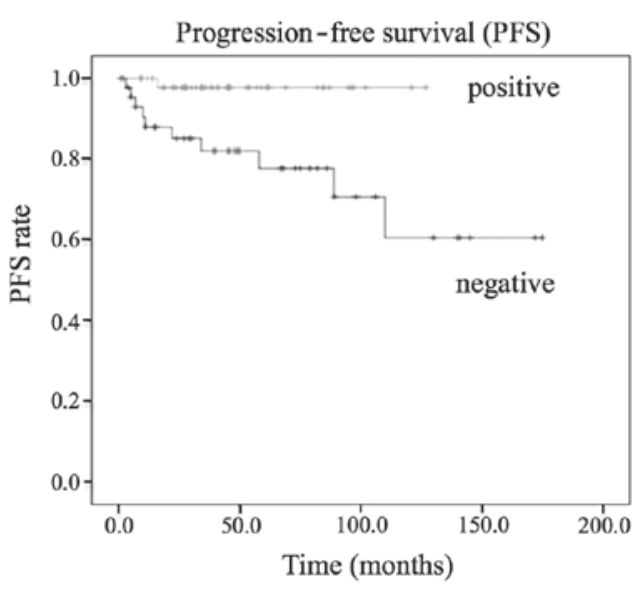

Log-rank test; $\mathrm{P}=\mathbf{0 . 0 0 5}$

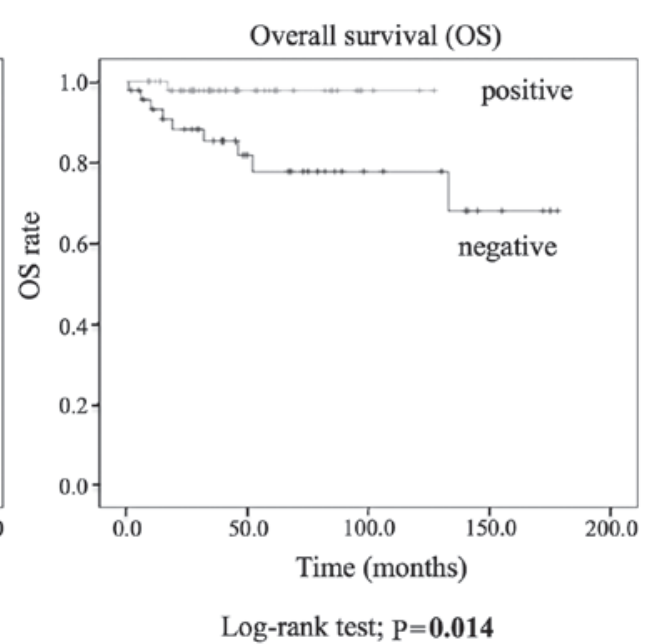

Log-rank test; $\mathrm{P}=\mathbf{0 . 0 1 4}$

Figure 2. Kaplan-Meier progression-free and overall survival curves showing the association between human leukocyte antigen class I expression patterns and prognosis in endometrial cancer.

Association between HLA class I expression pattern and clinicopathological factors. The HLA class I-negative pattern was significantly associated with advanced FIGO stage $(\mathrm{P}<0.001)$, LVSI $(\mathrm{P}=0.003)$ and lymph node metastasis $(\mathrm{P}=0.005)$ (Table I). However, there was no significant association between HLA class I expression and age, tumor grade, myometrial invasion or peritoneal cytology.

Association between HLA class I expression pattern and prognosis. A Kaplan-Meier analysis using the log-rank test revealed that patients with the HLA class I-positive pattern had significantly higher PFS $(\mathrm{P}=0.005)$ and $\mathrm{OS}(\mathrm{P}=0.014)$ rates compared with patients who had a negative pattern (Fig. 2).

Univariate and multivariate analyses for PFS and OS. In the univariate Cox regression analysis, a poor PFS was associated with advanced FIGO stage, invasion of more than half of the thickness of the myometrium (depth b), LVSI, tumor grade III, positive peritoneal cytology and HLA class I-negative pattern (Table III). In the multivariate analysis, only advanced 
Table IV. Cox regression analysis of overall survival.

\begin{tabular}{|c|c|c|c|c|}
\hline \multirow[b]{2}{*}{ Categories } & \multicolumn{2}{|c|}{ Univariate analysis } & \multicolumn{2}{|c|}{ Multivariate analysis } \\
\hline & HR $(95 \% \mathrm{CI})$ & P-value & HR $(95 \% \mathrm{CI})$ & P-value \\
\hline \multicolumn{5}{|l|}{ Age (years) } \\
\hline$>50$ vs. $<50$ & $2.329(0.296-18.317)$ & 0.424 & $0.984(0.108-8.946)$ & 0.989 \\
\hline \multicolumn{5}{|l|}{ FIGO stage } \\
\hline III/IV vs. I/II & $8.518(2.447-29.655)$ & 0.001 & $2.134(0.415-10.971)$ & 0.364 \\
\hline \multicolumn{5}{|l|}{ Myometrial invasion } \\
\hline Depth $a^{a}$ vs. $b^{b}$ & $3.387(0.990-11.588)$ & 0.052 & - & - \\
\hline \multicolumn{5}{|l|}{ LVSI } \\
\hline Positive vs. negative & $14.963(1.886-118.73)$ & 0.009 & $6.720(0.661-68.313)$ & 0.107 \\
\hline \multicolumn{5}{|l|}{ Tumor grade } \\
\hline 3 vs. $1 / 2$ & $7.792(1.647-35.760)$ & 0.002 & $2.928(0.71818 .363)$ & 0.134 \\
\hline \multicolumn{5}{|l|}{ Lymph node metastasis } \\
\hline Positive vs. negative & $6.544(1.709-25.054)$ & 0.006 & - & - \\
\hline \multicolumn{5}{|l|}{ Peritoneal cytology } \\
\hline Positive vs. negative & $0.035(<0.001-62.661)$ & 0.381 & - & 0.985 \\
\hline \multicolumn{5}{|c|}{ HLA class I expression pattern } \\
\hline Positive vs. negative & $0.114(0.014-0.913)$ & 0.041 & $0.482(0.044-5.254)$ & 0.466 \\
\hline
\end{tabular}

Bold print indicates statistical significance. Invasion of aless than half and ${ }^{\mathrm{b}}$ more than half of the thickness of the myometrium. HR, hazard ratio; CI, confidence interval; FIGO, International Federation of Gynecology and Obstetrics; LVSI, lymphovascular space involvement; HLA, human leukocyte antigen.

FIGO stage retained its significance as an independent prognostic factor of poor PFS (Table III).

In the univariate Cox regression analysis, poor OS was associated with advanced FIGO stage, positive LVSI, tumor grade 3 , positive lymph node metastasis and HLA class I-negative pattern (Table IV). In the multivariate analyses, none of the parameters examined was an independent prognostic factor for OS (Table IV).

\section{Discussion}

In this study, downregulation of HLA class I expression in endometrial cancer was found in $46.9 \%$ of the cases, which is similar to percentages previously reported in endometrial cancer patients [41\% (12) and 48\% (10)]. This frequency was also similar to several other types of cancer, including esophageal cancer (43\%), but lower compared with breast (67\%), lung $(70 \%)$, gastric $(75 \%)$, ovarian $(65 \%)$ and uterine cervical cancer $(81 \%)$ (4-11). The difference in frequency of the downregulation of HLA class I expression among several types of cancer may be due to the differences in organ-specific factors affecting HLA class I expression, such as immune responses $(13,17,18)$.

In the present study, downregulation of HLA class I expression was associated with advanced FIGO stage, LVSI and lymph node metastasis, which was consistent with previously reported results (10). It was also observed that HLA class I-negative patients exhibited lower PFS and OS compared with HLA class I-positive patients; this is consistent with previous findings suggesting that downregulation of HLA class I expression is associated with poor prognosis in a variety of cancers (4-11). The poor prognosis of patients with downregulation of HLA class I expression is considered to be due to a reduced host antitumor immune response, i.e., to the escape of tumor cells from T lymphocytes, as the presence of HLA class I antigens on the surface of tumor cells is considered a prerequisite for cytotoxic T-lymphocyte action $(13,18)$. In addition, the number of T lymphocytes was found to be significantly lower in tumors exhibiting downregulation of HLA class I expression compared with endometrial cancers with normal expression, suggesting that HLA class I molecules play an important role in the recruitment of T lymphocytes (13). An immunosuppressive enzyme, indoleamine 2,3-deoxygenase, has also been reported to be another prognostic factor in endometrial cancer (19). Although the detailed mechanism regulating tumor progression in endometrial cancer remains unclear, these results suggest that the immune system plays an important role in the progression of endometrial cancer.

Although the downregulation of HLA class I expression was found to be associated with poor OS in the univariate analysis, it was not identified as an independent factor in the multivariate analysis (Table IV). In addition, advanced FIGO stage was an independent predictive factor for worse PFS, but not for OS, in the multivariate analysis (Tables III and IV), which is consistent with the study of Bijen et al (10). These observations may be a consequence of the significant percentage of stage I patients in this study (80\%). This explanation is supported by the fact that the group exhibiting downregulation of HLA class I expression included more advanced-stage patients (10). 
It remains controversial whether HLA class I expression is a significant predictive prognostic factor in endometrial cancer. Downregulation of HLA class I expression was reported to be associated with worse disease-specific survival (13), whereas in other studies HLA class I expression was not found to be associated with clinicopathological parameters or survival (10). These different results, including our data, may be due to the different definitions of negative HLA class I expression in immunohistochemistry. Bijen et al (10) and the present study adopted strict criteria to classify negative HLA class I expression (defined as negative for both $\mathrm{HC} 10$ and $\beta 2-\mathrm{m})$. Further studies including a larger sample size and applying the same criteria to define HLA class I expression are required to elucidate the clinical implications of HLA class I expression in endometrial cancer.

In conclusion, downregulation of HLA class I expression was observed in half of endometrial cancers in the present study, similar to what has been reported in several other types of cancer. The downregulation of HLA class I expression was associated with tumor progression and poor PFS and OS. Therefore, HLA class I expression may be useful for predicting postoperative outcome in endometrial cancer and, thus, may be added to the existing well-known predictive prognostic factors, such as lymph node metastasis and LVSI.

\section{Acknowledgements}

This study was supported in part by the JSPS KAKENHI grants 23592425, 23791845 and 26462525 for Scientific Research from the Ministry of Education, Science and Culture, Japan.

\section{References}

1. Garrido F, Ruiz-Cabello F, Cabrera T, Pérez-Villar JJ, López-Botet M, Duggan-Keen M and Stern PL: Implications for immunosurveillance of altered HLA class I phenotypes in human tumours. Immunol Today 18: 89-95, 1997.

2. Hicklin DJ, Marincola FM and Ferrone S: HLA class I antigen downregulation in human cancers: T-cell immunotherapy revives an old story. Mol Med Today 5: 178-186, 1999.

3. Gabrilovich D and Pisarev V: Tumor escape from immune response: Mechanisms and targets of activity. Curr Drug Targets 4: 525-536, 2003.

4. Rolland P, Deen S, Scott I, Durrant L and Spendlove I: Human leukocyte antigen class I antigen expression is an independent prognostic factor in ovarian cancer. Clin Cancer Res 13: 3591-3596, 2007.

5. Mehta AM, Jordanova ES, Kenter GG, Ferrone S and Fleuren GJ: Association of antigen processing machinery and HLA class I defects with clinicopathological outcome in cervical carcinoma. Cancer Immunol Immunother 57: 197-206, 2008.
6. Speetjens FM, de Bruin EC, Morreau H, et al: Clinical impact of HLA class I expression in rectal cancer. Cancer Immunol Immunother 57: 601-609, 2008.

7. Ueda Y, Ishikawa K, Shiraishi N, Yokoyama S and Kitano S: Clinical significance of HLA class I heavy chain expression in patients with gastric cancer. J Surg Oncol 97: 451-455, 2008.

8. Mizukami Y, Kono K, Maruyama T, Watanabe M, Kawaguchi Y, Kamimura K and Fujii H: Downregulation of HLA class I molecules in the tumour is associated with a poor prognosis in patients with oesophageal squamous cell carcinoma. Br J Cancer 99: 1462-1467, 2008.

9. Kikuchi E, Yamazaki K, Torigoe T, Cho Y, Miyamoto M, Oizumi S, Hommura F, Dosaka-Akita $\mathrm{H}$ and Nishimura $\mathrm{M}$ : HLA class I antigen expression is associated with a favorable prognosis in early stage non-small cell lung cancer. Cancer Sci 98: 1424-1430, 2007.

10. Bijen CB, Bantema-Joppe EJ, de Jong RA, Leffers N, Mourits MJ, Eggink HF, van der Zee AG, Hollema H, de Bock GH and Nijman HW: The prognostic role of classical and nonclassical MHC class I expression in endometrial cancer. Int J Cancer 126: 1417-1427, 2010.

11. Kaneko K, Ishigami S, Kijima Y, et al: Clinical implication of HLA class I expression in breast cancer. BMC Cancer 11: 454, 2011.

12. Barrier BF, Kendall BS, Sharpe-Timms KL and Kost ER: Characterization of human leukocyte antigen-G (HLA-G) expression in endometrial adenocarcinoma. Gynecol Oncol 103: 25-30, 2006

13. de Jong RA, Boerma A, Boezen HM, Mourits MJ, Hollema H and Nijman HW: Loss of HLA class I and mismatch repair protein expression in sporadic endometrioid endometrial carcinomas. Int J Cancer 131: 1828-1836, 2012.

14. Murakami A, Fukushima C, Yoshidomi K, Sueoka K, Nawata S, Yokoyama Y, Tsuchida S, Ismail E, Al-Mulla F and Sugino N: Suppression of carbonyl reductase expression enhances malignant behaviour in uterine cervical squamous cell carcinoma: Carbonyl reductase predicts prognosis and lymph node metastasis. Cancer Lett 311: 77-84, 2011.

15. Torigoe T, Asanuma H, Nakazawa E, Tamura Y, Hirohashi Y, Yamamoto E, Kanaseki T, Hasegawa T and Sato N: Establishment of a monoclonal anti-pan HLA class I antibody suitable for immunostaining of formalin-fixed tissue: Unusually high frequency of down-regulation in breast cancer tissues. Pathol Int 62: 303-308, 2012.

16. Murakami A, Yakabe K, Yoshidomi K, Sueoka K, Nawata S, Yokoyama Y, Tsuchida S, Al-Mulla F and Sugino N: Decreased carbonyl reductase 1 expression promotes malignant behaviours by induction of epithelial mesenchymal transition and its clinical significance. Cancer Lett 323: 69-76, 2012.

17. Seliger B: Molecular mechanisms of MHC class I abnormalities and APM components in human tumors. Cancer Immunol Immunother 57: 1719-1726, 2008.

18. Kondratiev S, Sabo E, Yakirevich E, Lavie O and Resnick MB: Intratumoral $\mathrm{CD}^{+} \mathrm{T}$ lymphocytes as a prognostic factor of survival in endometrial carcinoma. Clin Cancer Res 10: 4450-4456, 2004.

19. Ino K, Yamamoto E, Shibata K, Kajiyama H, Yoshida N, Terauchi M, Nawa A, Nagasaka T, Takikawa $\mathrm{O}$ and Kikkawa F: Inverse correlation between tumoral indoleamine 2,3-dioxygenase expression and tumor-infiltrating lymphocytes in endometrial cancer: Its association with disease progression and survival. Clin Cancer Res 14: 2310-2317, 2008. 